\title{
Evaluation of the Efficacy of Baby Chick Ranikhet Disease Vaccine and Bangla Baby Chick Ranikhet Disease Vaccine in Fayoumi Chicks
}

\author{
Zafar Ahmed Bhuiyan ${ }^{1,2}$, Paritosh Kumar Biswas ${ }^{1 *}$, M Nural Anwar², Abdul Ahad ${ }^{1}$ and Nitish Chandra Debnath ${ }^{1}$ \\ ${ }^{1}$ Department of Microbiology, Chittagong Veterinary University, Pahartali 4202, Chittagong, Bangladesh, ${ }^{2}$ Department of Microbiology, University of \\ Chittagong, Chittagong 4331, Bangladesh
}

[Received 13 August 2006; Accepted 07 October 2006]

\begin{abstract}
A study was undertaken for testing the comparative efficacy of two live vaccines produced in Bangladesh to control Newcastle disease (ND) in chickens. One of these vaccines named 'baby chicks Ranikhet disease vaccine (BCRDV), a government vaccine produced by the Livestock Research Institute, Mohakhali, Dhaka, and the other named 'Bangla-BCRDV ${ }^{\circledR}$, , a commercial vaccine produced recently by a pharmaceutical company. Both the vaccines are prepared using the ' $F$ ' strain of Newcastle disease virus (NDV). Three hundred Fayoumi chicks distributed in 3 groups were used in this study. The results of this study revealed that, at the age of 49 days the survival rates of chicks belonged to BCRDV, Bangla-BCRDV ${ }^{\circledR}$ and control groups were 69, 11 and 18\%, respectively. The survival rate of Fayoumi chicks in the BCRDV group was significantly higher than that of the two other groups $(p<0.05)$. Conversely, almost similar survival rates of chicks were recorded in the control and Bangla-BCRDV ${ }^{\circledR}$ groups $(p>0.05)$. At day-1, the haemagglutination inhibition (HI) titre to NDV of the chicks was $\log _{2} 5.8 \pm 0.79$ (SEM). At day 35 the same HI titre was observed in the chicks of the BCRDV group, which was, 1 and $1.5 \log$ (base 2) higher than the chicks of the control and Bangla-BCRDV $^{\circledR}$ group, respectively. At day-49, HI titres to $\mathrm{NDV} \geq \log _{2} 12$ were recorded in the chicks of all the three groups that survived having challenged with velogenic NDV. There were no significant differences in weekly mean body-weights of the chicks in all the three groups until the week 8 when the mean bodyweight of the chicks was higher in the BCRDV group compared with the two others. These results indicted that the efficacy of BCRDV in Fayoumi chicks against ND was better than the Bangla-BCRDV ${ }^{\circledR}$.
\end{abstract}

Keywords: Baby chick ranikhet disease vaccine, Bangla baby chick ranikhet disease vaccine, Newcastle disease (ND), Newcastle disease virus (NDV), Newcastle disease vaccine (NDC), Fayoumi chicks

\section{Introduction}

Newcastle disease (ND), popularly known as 'Ranikhet disease' of birds in the Indian-subcontinent, is an epidemic and endemic disease in Bangladesh. The disease is considered one of the dominant constraints to poultry farming/rearing in all developing countries including Bangladesh. Every year, it causes epidemics in village chickens reared under the semi-scavenging and scavenging systems in rural areas of Bangladesh ${ }^{1-3}$. Also, there are reports in the country on its incidences in commercial chickens that are generally reared under improved bio-security along with the provision of vaccination against the disease ${ }^{4}$. Vaccine and/or vaccination failures are sometimes blamed for these unpleasant happenings.

The level of immunity in birds followed by ND-vaccination depends on various factors such as strains of the vaccine virus, titre of the viruses in the vaccines, routes and doses of administration, level of maternally derived antibodies in the blood serum and the presence or absence of other infections ${ }^{5}$. The higher the virulent vaccine-virus used for the vaccination against
ND the more antibodies it would generate, thus ensuring better protection $^{6}$. However, the stronger vaccine virus can produce the clinical disease if the chickens have not been primed with the less virulent virus. For serving both the purposes, the Livestock Research Institute (LRI), Mohakhali, Dhaka has been producing two live vaccines against ND, viz., 'baby chick Ranikhet disease vaccine' (BCRDV), which is F strain (a lentogenic strain) of Newcastle disease virus (NDV), and 'Ranikhet disease virus' (RDV), which is a more virulent mesogenic (Mukteswar) strain of the virus. The BCRDV is used for the chicks $>2$ months of age and RDV is only allowed for the adult birds $>2$ months of age that have been initially vaccinated with any ND-vaccine containing the lentogenic virus. Until recently, the LRI was the sole institute in Bangladesh producing the ND-vaccines and distributing throughout the country at subsidized rates via the district and Upazila Livestock Offices under the Department of Livestock Services. The quantity of the vaccines produced by the LRI is inadequate to meet up the country's growing demands for these vaccines. Recently, one pharmaceutical company (FnF Pharmaceuticals Ltd) has started producing the vaccine named,

*Corresponding author:

Dr. Paritosh Kumar Biswas, Professor \& Head, Department of Microbiology, Chittagong Veterinary University, Pahartali, Chittagong 4202, Bangladesh Tel (Office): (031) 659093/110, 126; Fax: +880 (031) 659492; E-mail: biswaspk2000@yahoo.com 
'Bangla-baby chick Ranikhet disease vaccine' (Bangla-BCRDV ${ }^{\circledR}$ ) by using the same F strain of NDV (as in BCRDV) in parallel to the LRI.

Christensen ${ }^{7}$ strongly recommended that the quality of vaccines produced from the LRI have to be checked and ensured. It is also questionable how the quality of Bangla-BCRDV ${ }^{\circledR}$ is maintained. If the Bangla-BCRDV ${ }^{\circledR}$ is proved to be of good quality it could be recommended as an alternative vaccine to BCRDV for the chickens reared in the rural areas of Bangladesh under different income generating programmes like Smallholder Livestock Development Project 2, which is being operated in 6 southern districts of Bangladesh. The objective of this study is to evaluate the efficacy of the two vaccines, viz., BCRDV and Bangla-BCRDV ${ }^{\circledR}$ against ND in the Fayoumi chickens.

\section{Materials and Methods}

Vaccines used

Two vaccines were used in this experiment, viz., BCRDV (produced by the LRI) and Bangla-BCRDV ${ }^{\circledR}$ (produced by FnF Pharmaceuticals Ltd). Both the vaccines are available in lyophilised forms and they are prepared using the same strain (F, a lentogenic virus) of NDV. The BCRDV was supplied only in 100 doses' glass-vials without any labelling on the vial; however, it could be identified by the green colour of its contents. In contrast, Bangla-BCRDV ${ }^{\circledR}$ were sold in 300, 500 and 1,000 doses' vials and on each vial was a brief description of the vaccine including the batch number, and the dates of its production and expiry. Also, a diluent was provided with the Bangla-BCRDV ${ }^{\circledR}$; distilled water was recommended for BCRDV instead of any specific diluents. Both vaccines could be administered intraoccularly.

\section{Chickens for the trail of the vaccines}

Three hundred Fayoumi day-old chicks were collected from the Pahartali Zonal Poultry Farm (PZPF), Chittagong, which is a government poultry farm. Generally, in this PZPF, Fayoumi and Rhode Island Red (RIR) breeds were maintained as two parental breeds to produce the Sonali (Crosbred; $F_{1}$ generation) chicks. The Sonali, and sometimes Fayoumi, chicks were supplied to the rural areas for been reared up by the poor people, particularly by the women, as a part of their income generating activities. Under different poverty amelioration programmes like the SLDP-2, rearing of higher egg-producing hens, especially Sonali and sometimes Fayoumi, were encouraged and facilitated by supplying microcredits. At day 1 , randomly selected 10 from the collected chicks were euthanased and sacrificed humanely to take blood sera from them. The remaining chicks were distributed in three groups: Group 1 and Group 3 comprising 125 birds each, and Group 2 was the control group having 40 chicks.

\section{Management of chicks}

The chicks distributed in three groups were reared in three poultry sheds (well-separated by fencing) of a newly constructed poultryhouse. The distance of the nearby poultry farm from this newly constructed house was about half a kilometre. Optimum space and ventilation was also ensured for each bird. The chicks under the three experimental groups were reared up for 120 days by supplying ample balanced feed and water. At day 5 and 21, the chicks belonged to Group 1 and Group 3 were vaccinated intraocculary twice with the BCRDV and Bangla BCRDV respectively, because the vaccine producers recommend this schedule. For Bangla BCRDV ${ }^{\circledR}$ pooled preparation of vaccine made from 3 vials of 3 different batches (batch No, 7, 8 and 9) was used. Since the BCRDV was supplied in vials without labelling, it was impossible to collect vaccine produced in different batches. Thus, in case of the BCRDV 3 vaccine, vials were collected from three Upazila livestock offices based at Kotowali, Pachlaish and Anowara Upazila to prepare a pooled vaccine. The chicks under the control group were given placebo. No other vaccines were given to any of the chicks during the course of this study.

\section{Challenge virus}

For this study, velogenic NDV was gratefully received from the Department of Microbiology, Chittagong Veterinary University, Chittagong. The virus was initially isolated from a Sonali hen reared in the SLDP-2 area. The mean embryo death time (MEDT) of the virus was $<48 \mathrm{~h}$. The titre of the virus in the collected sample was done in the embryonated chicken-eggs following the method described by Reed and Muench ${ }^{8}$. The dilution at $10^{-7.4}$ contained the virus titre $100 \log \mathrm{ELD}_{50}$ (embryo lethal dose 50). This dilution was used as challenge inoculum. At day 42, all chicks were administered, by using a micro-titration pipette, orally with this inoculum @ 0.2 ml per chick.

\section{Measures applied for testing the efficacy of the vaccines}

Three measures were applied to compare the efficacy of the two vaccines in Fayoumi chicks including the estimation of survival rates of the chicks belonged to each group, and the level of haemagglutination-inhibition (HI) titre to NDV in blood sera of the chicks and gaining of body-weight at weekly intervals. After the challenge given with the velogenic NDV, the number of total deaths (at weekly intervals) of the chicks in each group was recorded. A death that had been attributed to Newcastle disease (ND) was considered as 'failure' and the deaths encountered due to non-specific reasons were 'censored'. Survival rates of each of the groups were calculated considering occurrence of the ND as Failure. For diagnosing a case of failure organ samples such as brain, spleen, trachea and lungs were collected from a dead chick. Virological inoculum was prepared from these pooled organs. For each inoculum prepared, at least four 9 to 10-day-old embryonated chicken eggs were inoculated through the allantoic sac route with $0.2 \mathrm{ml}$ inoculum/egg and incubated at $37^{\circ} \mathrm{C}$. These inoculated eggs were checked everyday for up to 5 days for embryo-mortality. Embryos that died after $24 \mathrm{~h}$ were discarded because these deaths could be resulted from non-specific reasons. Allantoic fluids were only harvested from the eggs when the embryos died after $24 \mathrm{~h}$ but before $120 \mathrm{~h}$. These harvested allantoic fluids were tested for 
the presence of haemagglutinating virus using $1 \%$ chicken red blood cells (RBC). Finally, the presence of the NDV in this fluid was determined by specific haemagglutination inhibition (HI) test. To do the HI test hyperimmune serum to the NDV with HI titre $\log _{2} 13$ (raised in a hen) was used. For negative and positive control uninfected allantoic fluid and F strain of NDV was respectively used.

Blood was collected aseptically from 10 chicks from the brachial vein of each group at day 35 and 49. From the individual blood sample serum was separated and collected in an Eppendorf tube (2.5-ml). All of the serum samples were kept frozen at $-85^{\circ} \mathrm{C}$ until analysed. The HI titre to the NDV in each serum sample was determined by $\mathrm{HI}$ test, done according to the recommended procedure of $\mathrm{OIE}^{9}$. For this test, 4 haemagglutination unit (4 HAU) of $F$ strain of the NDV was used. For a negative control specific pathogen-free chicken serum supplied by the Veterinary Laboratories Agency, Surrey (UK) was used. For a positive control, pooled serum taken from 5 Fayoumi hens (reared at PZPF) that had been vaccinated with the RDV three weeks before was used. At least $20 \%$ of the chicks of the three experimental groups were weighted at weekly intervals.

\section{Data analysis}

All data were entered into the spread-sheet programme (Excel, Microsoft Co) for data summary and statistical analysis. The differences in the level of $\mathrm{HI}$ titres and gaining of weekly bodyweight of the three groups of chicks were calculated using the Student's $t$ test. The differences of the survival rates of the chicks of these three groups were shown using a $\chi^{2}$ test.

\section{Results}

Figure 1 shows the weekly survival rates of Fayoumi chicks in the three experimental groups. Up to the $5^{\text {th }}$ week of age the survival rates of the chicks belonged to all of the three groups were almost $100 \%$. Having challenged with the wild velogenic NDV, most of the chicks in the Bangla-BCRDV ${ }^{\circledR}$ and control groups started showing clinical symptoms (like chalky white diarrhoea, offfeeding, ruffled feather, huddling together and twisting of the neck) by $24 \mathrm{~h}$.A few birds in the BCRDV group also showed these symptoms. After $48 \mathrm{~h}$ (in the $2^{\text {nd }}$ day) of the challenge given massive mortalities happened in the Bangla-BCRDV ${ }^{\circledR}$ and the control group; comparatively lower mortality observed in the BCRDV group. Samples collected from all of the cases of deaths revealed the presence of NDV, confirming the occurrences of failures; consequently, the survival rates of the chicks declined to 26 and $63 \%$ to Bangla-BCRDV ${ }^{\circledR}$ and the control group, respectively by the $7^{\text {th }}$ week. However, the difference of the survival rates between these two groups was not statistically significant $(p>0.5)$. Compared with these two groups, a significant higher survival rate of chicks was recorded in the BCRDV group by then $(p<0.5)$. There were 2,2 and 1 clinically sick birds belonged to the Bangla-BCRDV ${ }^{\circledR}$, the BCRDV and the control groups survived even up to the $8^{\text {th }}$ week but they developed neurological syndrome (particularly torticollis) and finally all of them succumbed to death at the end of $8^{\text {th }}$ week, resulting the survival rates of the chicks of these three groups 11,69 and $18 \%$, respectively.

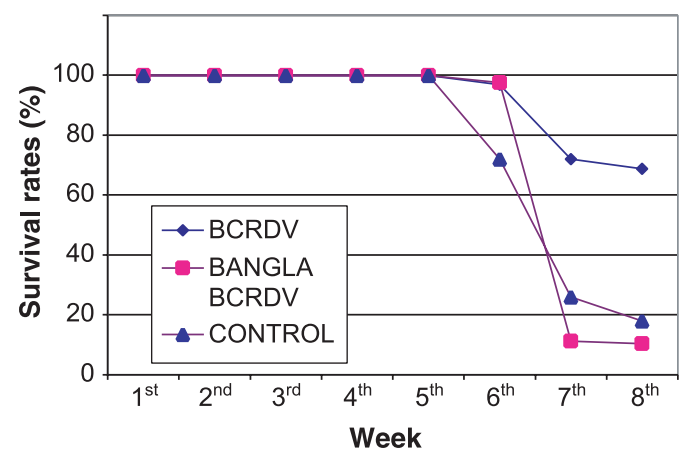

Figure 1. Survival rates of two groups of Fayoumi chicks that had been vaccinated with the BCRDV and Bangla-BCRDV compared with the un-vaccinated control group. $B C R D V=B a b y$ chicks Ranikhet disease vaccine

At day 1 , the mean $\log _{2} \mathrm{HI}$ titre in blood sera of the experimented chicks to NDV was 5.8, which was the level of maternally derived antibodies to NDV (Table 1). At day 35, the HI titre to NDV in the chicks of the control group was $\log _{2} 4.5$, which was $1.5(p>0.05)$ but only $0.5(p<0.5) \log$ lower than that of the BCRDV and Bangla$\mathrm{BCRDV}^{\circledR}$ group, respectively. After 7 days or at the age of 49 day of the challenge given with wild velogenic NDV, the level of HI titre however increased sharply and was $\geq \log _{2} 12$ in the surviving chicks of all of the three groups.

Table 1. Temporal dynamics of $\log _{2}$ haemagglutination inhibition (HI) titres to the Newcastle disease vaccine (NDC) of Fayoumi chicks distributed in three groups under a vaccination trail: Group 1 for the BCRDV, Group 2 for the control group, and Group 3 for Bangla-BCRDV

\begin{tabular}{lccc}
\hline Fayoumi chick group & \multicolumn{3}{c}{ Age of chicks } \\
\cline { 2 - 4 } & At day $1^{*}$ & At day 35 & At day 49 \\
\hline 1 - BCRDV & $5.8 \pm 0.79$ & $5.8 \pm 0.42^{\mathrm{a}}$ & $12.8 \pm 1.69^{\mathrm{a}}$ \\
2 - Control & $5.8 \pm 0.79$ & $4.53 \pm 0.55^{\mathrm{b}}$ & $14.0 \pm 0^{\mathrm{a}}$ \\
3 - Bangla BCRDV & $5.8 \pm 0.79$ & $5.0 \pm 0^{\mathrm{c}}$ & $12.0 \pm 0^{\mathrm{a}}$ \\
\hline
\end{tabular}

${ }^{*}$ The level of maternally derived HI antibodies in blood sera to the NDV at that time. Only different superscripted alphabets between two rows in a column indicate significant difference $(p<0.5)$. BCRDV = Baby chicks Ranikhet disease vaccine

The weekly mean body-weights of the chicks in the three groups are shown in Table 2. At day 1, the mean body-weight was about $30 \mathrm{~g}$. The weekly mean body-weight of the chicks observed from the first week to the $7^{\text {th }}$ was almost equal among the three groups $(p>0.05)$. For the $8^{\text {th }}$ week, higher mean body-weight was recorded in the chicks of the BCRDV group compared with two other groups $(p>0.05)$ Bangla-BCRDV ${ }^{\circledR}$ and the control; in the later two groups the mean body weight was however identical $(p<0.5)$. 
Bhuiyan et al.

Table 2. Arithmetic mean ( \pm SEM) weight (in g) of Fayoumi chicks at weekly intervals during the experimental trial of a government and a commercially produced Newcastle disease vaccine (NDC) in Bangladesh

\begin{tabular}{|c|c|c|c|c|c|c|c|c|}
\hline $\begin{array}{l}\text { Fayoumi chick } \\
\text { group }\end{array}$ & \multicolumn{8}{|c|}{ Weight of chicks at weekly intervals (g) } \\
\hline 1 - BCRDV & $30.1 \pm 1.3^{\mathrm{a}}$ & $129 \pm 15.2^{\mathrm{a}}$ & $195 \pm 26.4^{\mathrm{a}}$ & $298 \pm 37.5^{\mathrm{a}}$ & $371 \pm 33.8^{\mathrm{a}}$ & $474 \pm 58.3^{\mathrm{a}}$ & $542 \pm 62.5^{\mathrm{a}}$ & $506 \pm 100.7^{a}$ \\
\hline 2 - Control & $29.8 \pm 1.5^{\mathrm{a}}$ & $144 \pm 5.5^{\mathrm{a}}$ & $198 \pm 4.5^{\mathrm{a}}$ & $292 \pm 19.3^{\mathrm{a}}$ & $374 \pm 44.5^{\mathrm{a}}$ & $464 \pm 41.0^{\mathrm{a}}$ & $522 \pm 30.3^{\mathrm{a}}$ & $474 \pm 76.4^{b}$ \\
\hline 3 - Bangla BCRDV & $29.6 \pm 1.4^{\mathrm{a}}$ & $142 \pm 11.4^{\mathrm{a}}$ & $194 \pm 17.8^{\mathrm{a}}$ & $279 \pm 38.7^{\mathrm{a}}$ & $362 \pm 46.0^{\mathrm{a}}$ & $486 \pm 33.4^{\mathrm{a}}$ & $519 \pm 27.7^{\mathrm{a}}$ & $424 \pm 66.5^{\mathrm{b}}$ \\
\hline
\end{tabular}

Only different superscripted alphabets between two rows in a column indicate significant difference $(p<0.5)$. BCRDV = Baby chicks Ranikhet disease vaccine

\section{Discussion}

The results of the present vaccination trail on the BCRDV and Bangla-BCRDV ${ }^{\circledR}$ revealed that the BCRDV was far more effective than the Bangla-BCRDV to protect the Fayoumi chicks from the velogenic strain of Newcastle disease vaccine (NDC). Kafi ${ }^{10}$, however, reported that both the BCRDV and Bangla-BCRDV produced more or less the same HI antibodies titre to NDV in chicks when they had been vaccinated with these two vaccines; this means, both the vaccines could have given the same protection if they were challenged with velogenic NDV. They did not study this. In the present study, at day 35, the HI titre to the NDV induced by the vaccine Bangla-BCRDV was almost $1 \log _{2}$ lower than the level generated by the BCRDV. The differences in results obtained in these two studies can be explained that they were conducted in different types of chickens with two distinct chick-management profiles and by collecting the vaccines from different batches of their production.

The aim of this study was to verify the efficacy of the BCRDV and Bangla-BCRDB ${ }^{\circledR}$ in the Fayoumi chicks by following the exact instructions as given by their producers, the LRI and the FnF Pharmaceuticals Ltd. That was the reason why we did not deliberately measure the virus titres in these two vaccines before going to vaccinate the chicks with these. However, at the age of 35 days, the titre of HI antibodies to the NDV induced by the BanglaBCRDV in the Fayoumi chicks was at the level of protective threshold $\left(\log _{2} 5\right)$, which declined further owing to their catabolism, rendering the chicks immunologically deficit to protect them for more 25 days (until a stronger vaccine - the RDV was supposed to be used at the age of 60 days) from the invasion of the wild velogenic the NDV circulating ${ }^{11}$ in the SLDP-2 areas. It is speculated that insufficient titre of the virus in the Bangla-BCRDV could be one of the reasons for generating inadequate antibody titre.

To our knowledge it was the first time in Bangladesh the efficacy testing of Newcastle disease vaccine (NDV) was done by measuring the survival rates of chicks (after vaccination and challenge) and calculating the occurrences of Newcastle disease as contributory causes of the deaths of chicks as failures. One of the limitations of this study was the inability to investigate the shedding-time of the vaccinated virus in the faeces due to insufficient molecular facilities to identify small amount of viral nucleic acid in the faeces.
Although the HI titre in the chicks of the control group was lower than the two other experimental groups, these chicks had some antibodies (at day 35 the $\mathrm{HI}$ titre was $\log _{2} 4.5$ ). Investigation to the parent farm (PZPF), from where the chicks were collected, revealed that the Farm management used the killed-vaccine (Imopest) twice against Newcastle disease at day 12 and day 120. The higher maternally derived antibody (MDA) to NDV at day 1 might have been linked to this killed-vaccine given. The presence of $\mathrm{HI}$ antibodies in control chicks at day 35 indicated that these chicks had the scopes to become either persistently infected with the NDV or exposed to the virus or its antigen. Because the chicks of the three groups were attended by the same attendant there was a possibility that some chicks of the control group could have exposed to the vaccine virus or its antigen, through the attendant showing some antibodies to the NDV.

The chicks were challenged with the wild velogenic NDV at the age of 42 day. However, in the week 8 there was a difference (Table 2) found in the mean body-weight of the surviving chicks between the BCRDV and the two other severely affected groups. Because majority of the chicks of the BCRDV group was clinically healthy and they fed normally than the clinically affected chicks in the Bangla-BCRDV and the control groups, the mean bodyweight of the survivors in the BCRDV was higher in the subsequent week.

The survival rate of the chicks in the BCRDV group was $68 \%$, that means $32 \%$ chicks in this group also died from Newcastle disease when a challenge was given with the wild velogenic strain of NDV isolated from the SLDP-2 area ${ }^{12}$. So it can be deduced that the government vaccine, the BCRDV, produced from the lentogenic strain of the NDV (F strain) might not be enough to protect all the chicks even for up to 42 days despite the chicks had been given booster vaccination with the BCRDV at day 21 following the recommendation of the manufacturer. To protect the chicks of the PZPF from Newcastle disease for at least 2 months by using only the BCRDV the present practicing vaccinationregimen might be revised with the provision of applying the vaccine three times - at the first week, day 21 and day 40-42 instead of twice before giving the more potent vaccine, i.e., the RDV (Mukteswar strain of RDV) at day 60. Alternatively, the RDV could be given at day 40-42 after having the chicks primed with the BCRDV at the first week of their age followed by at day 21. Because 
this vaccine strain is more virulent it would produce more antibodies to protect the chicks from the velogenic virus ${ }^{6}$. However, before going to introduce this proposed schedule studies are further needed.

Based on the results of this study we could not recommend the Bangla-BCRDV as an alternative vaccine to the BCRDV for the SLDP-2 areas. We also realize that just one study is not enough to make a concluding remark on the efficacy of a commercial vaccine; more studies are needed in different situations because vaccine failures and suboptimal functioning are not only related with a vaccine itself; but the process of vaccination, the management of the chicks and the vaccine-recipients' immunological incompatibilities - all might have influences over these unexpected consequences. Christensen ${ }^{7}$ recommended setting of quality assurance for the vaccines produced from the LRI, Bangladesh. Presently, it is in dark how the commercial companies engaged in the production of vaccines for animalusages; whether they comply with the quality and safety issues is not clear. Therefore, a government-based regulatory system should be active on them.

\section{Acknowledgement}

This study is a part of an on going MPhil (in Microbiology) programme being undertaken at the Department of Microbiology, University of Chittagong and the Department of Microbiology, Chittagong Veterinary University, Chittagong financed by the DANIDA-SLDP-2 Fellowships. We thank the financing agencies for supporting the study.

\section{References}

1. Barman LR. 2002. An epidemiological and experimental study of Newcastle disease in village chickens of Bangladesh. MSc Thesis. The Royal Veterinary \& Agricultural University, Dyrlaegevej 2, Frederiksberg C, Denmark.

2. Biswas PK. 2003. Epidemiological scenario and quantification of the semi-scavenger losses attributed to main endemic disease of poultry. Final Report, pp 81. Chittagong Government Veterinary College, Pahartali, Chittagong, Bangladesh.
3. Chowdhury SI, Chowdhury TIMFR, Sarker AJ, Amin MM \& Hossain WIMA. 1982. Studies on Newcastle disease in Bangladesh: The role of residual maternal antibody on immune response and selection of an optimum age for primary vaccination of chicks. A Research Report, pp 12-22. Bangladesh Agricultural University, Mymensing, Bangladesh.

4. Islam MA, Ito T \& Kida H. 1998. Comparison of the haemagglutinin pattern and pathotypic activities of some Japanese isolates of Newcastle disease virus with reference strains. Bangladesh Vet J. 29: 1-8.

5. Beard CW, Villegas P \& Gilsson JR. 1993. Comparative efficacy of the $\mathrm{B}$ and VG/GA vaccine strains against velogenic viscerotropic Newcastle disease virus in chickens. Avian Dis. 37: 222-225.

6. Alexander DJ. 1880. Avian paramyxovirus. Vet Bull. 50: 737-752.

7. Christensen JP. 1998. Disease as a risk factor in relation to the rural poultry model in Bangladesh. In Poultry as a Tool in Poverty Eradication and Promotion of Gender Equity (Dolberg F \& Peterson PH eds), pp 188-197. Proceedings of a workshop on Poultry as a Tool in Poverty Eradication and Promotion of Gender Equity, 22 ${ }^{\text {nd }}$ $26^{\text {th }}$ March 1999. FAO Corporate Document Repository. The Danish Agricultural \& Rural Development Advisers' Forum, Yune Landboskole, Denmark.

8. Reed LJ \& Muench LH. 1938. A simple method of estimating fifty percent endpoint. Am J Hyg. 27: 493-497.

9. OIE. 2002. Newcastle disease. In International Animal Health Code, Chapter 2.1.15. Office International des Epizootics (OIE). Available at: http://www.oie.int. Accessed July 14, 2005.

10. Kafi MA, Rahman MB, Amin MM, Islam MR, Rahman MM \& Rahman MK. 2003. Comparative serological responses and protection conferred by vaccination with $\mathrm{V}_{4} \mathrm{HR}$ and BCRDV in chickens. Bangladesh J Vet Med. 1(1): 25-27.

11. Biswas PK. 2004. A longitudinal study to identify the causes on the mortality of 'Sonali' birds and broody hen chicks of key beneficiaries in the SLDP-2 area. SLDP-2 Applied Research Project Final Report, pp 78. Chittagong Government Veterinary College, Pahartali, Chittagong, Bangladesh.

12. Biswas PK, Uddin GMN, Barua H, Roy K, Biswas D, Ahad A \& Debnath NC. 2006. Immune status of semi-scavenging Sonali chickens in Bangladesh against Newcastle disease. Livestock Research for Rural Development (LRRD) 18, Article \# 74. Available at: http:// www.cipav.org.co/Irrd/Irrd186/bisw18074.htm. Accessed October 15, 2006. 